\title{
Once-daily tacrolimus in living donor liver transplant recipients
}

\author{
Yasuhiko Sugawara $^{1, *}$, Yoichi Miyata ${ }^{1}$, Junichi Kaneko" ${ }^{1}$, Sumihito Tamura ${ }^{1}$, Taku Aoki ${ }^{1}$, \\ Yoshihiro Sakamoto ${ }^{1}$, Kiyoshi Hasegawa ${ }^{1}$, Noriyo Yamashiki ${ }^{2}$, Norihiro Kokudo ${ }^{1}$ \\ ${ }^{1}$ Artificial Organ and Transplantation Division, Department of Surgery, Graduate School of Medicine, The University of \\ Tokyo, Tokyo, Japan; \\ ${ }^{2}$ Organ Transplantation Service, The University of Tokyo, Tokyo, Japan.
}

\begin{abstract}
Summary Once-daily tacrolimus (denoted here simply as OD) is a recently developed extendedrelease drug formulation. The purpose of the present study was to pharmacokinetically evaluate tacrolimus exposure and determine the feasibility of its de novo use in liver transplant recipients in the perioperative period. This was an open-label, single center study. Eligible patients were 18 to 65 years of age in the perioperative period after a liver transplant. Patients were initially treated with intravenous tacrolimus and then converted to the 10× milligram-for-milligram daily dose of OD administered once daily. Twenty-fourhour pharmacokinetic profiles were obtained on day 7 after the conversion. Laboratory and safety parameters were also evaluated. A total of 9 patients received OD, were successfully converted, and provided pharmacokinetic profiles. Intravenous tacrolimus and $\mathrm{OD}$ resulted in similar areas under the curve for $24 \mathrm{~h}\left(\mathrm{AUC}_{0-24}\right)$ of tacrolimus. OD was well tolerated with a safety profile comparable to that of intravenous tacrolimus. The $\mathrm{AUC}_{0-24}$ correlated with the minimum concentration of OD $(R=0.49)$. Renal and liver functions remained stable. None of the patients experienced acute rejection during the observation period. OD and intravenous tacrolimus provide equivalent drug exposure, allowing conversion of selected liver transplant recipients from intravenous tacrolimus to OD in the peri-operative period.
\end{abstract}

Keywords: Once-daily, donor, living donor liver transplantation, tacrolimus

\section{Introduction}

A twice-a-day tacrolimus formulation (TAC, Prograf ${ }^{\circledR}$, Astellas Pharmaceutical Corporation, Tokyo, Japan) is commonly used to prevent organ rejection in allogeneic kidney, liver, and heart transplant recipients (1). Medication compliance after transplantation, however, is a serious problem (2); a once-daily regimen could potentially improve compliance while maintaining safety. A once-daily tacrolimus formulation (OD) (3) that has the similar level of absorption as TAC with a reduced peak was recently developed and might help to

\footnotetext{
*Address correspondence to:

Dr. Yasuhiko Sugawara, Artificial Organ and Transplantation Surgery Division, Department of Surgery, Graduate School of Medicine, The University of Tokyo, 7-3-1 Hongo, Bunkyo-ku, Tokyo, 113-8655, Japan.

e-mail: yasusuga-tky@umin.net
}

resolve problems with compliance.

The present study reports the pharmacokinetics of tacrolimus during conversion from intravenous tacrolimus to OD in liver transplant recipients in the perioperative period. The aim of the present study was to determine the safety and tolerability of OD in these patients.

\section{Patients and Methods}

From February 2009 to May 2010, this institution performed 12 living donor liver transplants (LDLTs) and all of the patients involved were enrolled in this study. The recipients consisted of 5 men and 7 women with a median age of 49 years (range: 37-61 years). The median Model for End-stage Liver Disease score was 16 (range: 3-33). LDLT was indicated in cases of virus-related cirrhosis with or without hepatocellular carcinoma $(n=9)$ and cholestatic disease $(n=3)$.

The surgical technique for LDLT and the process 
of donor selection and evaluation have been described elsewhere $(4,5)$. Similar to the majority of liver transplantation centers worldwide (6), this institution uses a tacrolimus-based immunosuppression regimen. All of patients included in the present study initially received the same immunosuppressive regimen with TAC and methylprednisolone (7). In brief, TAC was administered by continuous intravenous infusion at a dose of $2.5 \mathrm{ug} / \mathrm{kg} / \mathrm{h}$ just after surgery. After the whole blood level of tacrolimus reached $17-18 \mathrm{ng} / \mathrm{mL}$, the dose was adjusted so that this level was maintained during the first week after surgery. Intravenous methylprednisolone was started during surgery (20 $\mathrm{mg} / \mathrm{kg} /$ day) and gradually tapered afterwards. When gastrointestinal function returned, OD and steroids were given orally. Steroid treatment was not discontinued in any of the patients.

Serial collection of whole blood samples to evaluate the pharmacokinetic profiles was performed at 0 (pre-dose), 1, 2, 3, 5, 10, and $24 \mathrm{~h}$ on day 7 after conversion to OD. On the other days, blood samples were obtained every 12 hours. Whole blood samples for pharmacokinetic analysis were frozen $\left(-20^{\circ} \mathrm{C}\right)$ until they were shipped to a central facility, where they were analyzed using a validated liquid chromatography/ tandem mass spectrometry assay (lower limit of quantification $0.1 \mathrm{ng} / \mathrm{mL}$ ). Whole blood tacrolimus concentrations below the lower limit of quantification were assigned a value of zero for calculation of the derived pharmacokinetic parameters. The area under the curve of the blood concentration-time curve (ng $\times \mathrm{h} / \mathrm{mL})$ from 0 to $24 \mathrm{~h}\left(\mathrm{AUC}_{0-24}\right)$ after dosing was calculated using the linear trapezoidal rule. Minimum concentrations $\left(\mathrm{C}_{\min } ; \mathrm{ng}\right.$, trough) were determined based on the observed whole blood tacrolimus concentration at $24 \mathrm{~h}$ on days when pharmacokinetic profiles were obtained. Total clearance $\left(\mathrm{CL}_{\text {tot }}\right)$, oral clearance $\left(\mathrm{CL}_{\mathrm{o}}\right)$, and bioavailability (BA) were calculated as follows:

$$
\begin{aligned}
& \mathrm{CL}_{\text {tot }}=\text { Doses of intravenous tacrolimus/AUC }(1 / \mathrm{h}) \\
& \mathrm{CL}_{\mathrm{o}}=\text { Doses of OD/AUC }(1 / \mathrm{h}) \\
& \mathrm{BA}=\mathrm{CL}_{\mathrm{tot}} / \mathrm{CL}_{\mathrm{o}}(\%)
\end{aligned}
$$

All adverse events and all serious adverse events were recorded during the pharmacokinetic study. Rejection episodes, all serious adverse events, and the following protocol-defined adverse events were recorded: post-transplant diabetes mellitus, hyperlipidemia, hypertension, infection (viral, bacterial, and fungal), renal dysfunction (serum creatinine level $\geq 2.0 \mathrm{mg} / \mathrm{dL}$ ), hepatic dysfunction, tremor, malignancy, and adverse events leading to study drug dose changes or study drug discontinuation. The incidence of biopsyconfirmed acute rejection and patient and graft survival were assessed throughout the study. Safety was assessed based on the incidence of adverse events and the results of routine clinical laboratory tests and recorded vital sign measurements.

Statistical analysis was performed using JMP 8.2 computer software (SAS Inc., Cary, NC). JMP 5.1 was used to perform one-way analysis of variance and $t$-tests. $P$ values of less than 0.05 were considered statistically significant for $t$-tests and $p$ values of less than 0.01 were considered statistically significant for analysis of variance with Bonferroni's correction (in comparison to preoperative levels).

\section{Results and Discussion}

In three patients, OD was started 10, 12, and 18 days after LDLT, but the trough level did not reach the target level and the study was stopped.

In the remaining 9 patients, intravenous tacrolimus was successfully converted to OD for an average of 9 days (range: 7-20 days) after LDLT. The dose of intravenous tacrolimus just before the conversion was $9.6 \times 10^{-3} \mathrm{mg} / \mathrm{kg} /$ day $(0.64 \mathrm{mg} /$ day $)$. The blood concentration of tacrolimus after conversion and the pharmacokinetic parameters are shown in Figure 1 and Table 1. Intravenous tacrolimus and OD resulted in a similar $\mathrm{AUC}_{0-24}$ of tacrolimus. OD was well tolerated with a safety profile comparable to that of intravenous tacrolimus. The $\mathrm{AUC}_{0-24}$ correlated with the $\mathrm{C}_{\min }$ for $\mathrm{OD}$ $(R=0.49)$ (Figure 2). Renal and liver functions remained stable. None of the patients experienced acute rejection during the observation period.

There were no adverse effects (post-transplant diabetes mellitus, hyperlipidemia, hypertension, renal dysfunction, or hepatic dysfunction) or acute rejection episodes during the observation period.

Overall, OD was well tolerated in this study and the adverse event profile was consistent with that of oral tacrolimus. In addition, serum creatinine levels remained stable after conversion to OD. There were no new cases of posttransplant diabetes or glucose intolerance and there was no increase in adverse events associated with tacrolimus use after conversion to OD. None of the patients experienced a biopsy-confirmed acute rejection episode during the pharmacokinetic study. Finally, there were no changes in the use of concomitant medication, including adjunctive immunosuppressive agents, during this study.

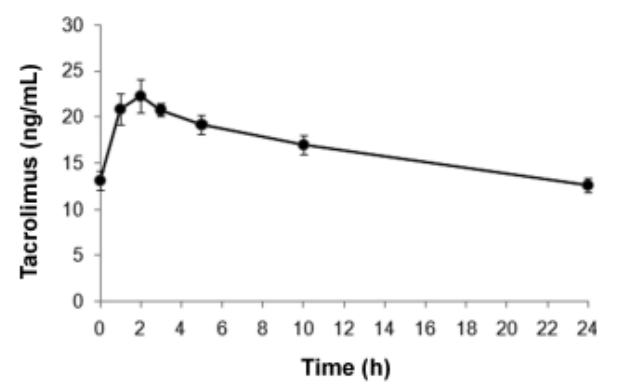

Figure 1. Blood concentration of tacrolimus after conversion. 
Table 1. Pharmacokinetic parameters $(n=9)$

\begin{tabular}{|c|c|c|}
\hline & Intravenous tacrolimus & OD \\
\hline Dose/BW (mg/kg) & $0.0105 \pm 0.0021$ & $0.124 \pm 0.047$ \\
\hline $\mathrm{C}_{\min } /$ Dose $/ \mathrm{BW}([\mathrm{ng} / \mathrm{mL}] /[\mathrm{mg} / \mathrm{kg}])$ & $1,468 \pm 321$ & $118 \pm 57$ \\
\hline $\mathrm{C}_{\max } /$ Dose $/ \mathrm{BW}([\mathrm{ng} / \mathrm{mL}] /[\mathrm{mg} / \mathrm{kg}])$ & - & $223 \pm 80$ \\
\hline $\mathrm{AUC}_{0-24}([\mathrm{ng} * \mathrm{~h} / \mathrm{mL}] /[\mathrm{mg} / \mathrm{kg}])$ & $35,232 \pm 7,702$ & $3,706 \pm 1,635$ \\
\hline Clearance $/ \mathrm{BW}(\mathrm{mL} / \mathrm{h} / \mathrm{kg})$ & $\mathrm{CL}_{\mathrm{tot}}=29.7 \pm 7.1$ & $\mathrm{CL}_{\mathrm{o}}=316 \pm 128$ \\
\hline Bioavailability (\%) & $10.6 \pm 4.0$ & - \\
\hline
\end{tabular}

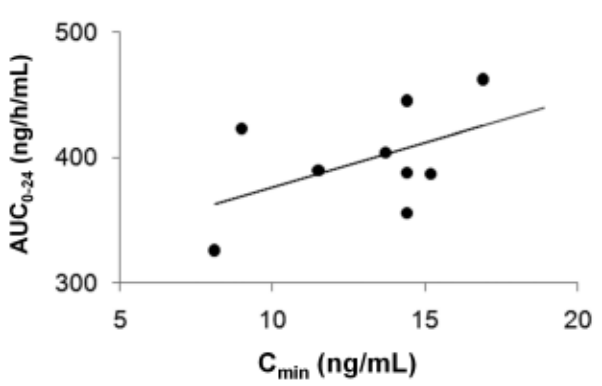

Figure 2. Correlation between AUC and $\mathrm{C}_{\text {min }}$. Correlation was expressed as $\mathrm{AUC}=7.1018 \times \mathrm{C}_{\min }+305.09 .\left(R^{2}=0.24\right.$, $p=0.17$ )

The level of steady-state intravenous tacrolimus exposure and $10 \times$ dose of OD were equivalent in perioperative liver transplant recipients regardless of sex or the presence of diabetes at the time of transplantation. The high correlation of exposure to trough levels for $\mathrm{OD}$ indicates that the system for monitoring TAC can be effectively used with patients receiving OD. There was significantly less intra-subject variability in tacrolimus exposure after conversion to OD.

The new TAC formulation provides a convenient, once-daily dosing option (8). Therapeutic regimens for transplant recipients are often complex and thus contribute to a high incidence of medication noncompliance and increased mortality and morbidity, including late acute rejection, late graft loss, and development of chronic rejection. Compliance is reported to increase from $59 \%$ with three-times-aday dosing regimen to $83 \%$ with once-daily dosing regimens, suggesting that administering fewer doses is a simple and effective way to improve compliance (9). The introduction of a once-daily dosing formulation may prove to be a valuable addition to the treatment armamentarium for transplant recipients.

\section{Conclusion}

In conclusion, liver transplant recipients in the perioperative period can be safely converted from intravenous tacrolimus to a $10 \times$ milligram-for-milligram daily dose of OD in the morning. A once-daily dosing regimen of tacrolimus can improve patient compliance while maintaining effective immunosuppression.

\section{Acknowledgements}

This study was supported by a grant-in-aid for Scientific Research from the Ministry of Education, Culture, Sports, and Science of Japan.

\section{References}

1. Vincenti F, Jensik SC, Filo RS, Miller J, Pirsch J. A longterm comparison of tacrolimus (FK506) and cyclosporine in kidney transplantation: Evidence for improved allograft survival at five years. Transplantation. 2002; 73:775-782.

2. Butler JA, Roderick P, Mullee M, Mason JC, Peveler RC. Frequency and impact of nonadherence to immunosuppressants after renal transplantation: A systematic review. Transplantation. 2004; 77:769-776.

3. Florman S, Alloway R, Kalayoglu M, Lake K, Bak T, Klein A, Klintmalm G, Busque S, Brandenhagen D, Lake J, Wisemandle K, Fitzsimmons W, First MR. Conversion of stable liver transplant recipients from a twice-daily Prograf-based regimen to a once-daily modified release tacrolimus-based regimen. Transplant Proc. 2005; 37:1211-1213

4. Kokudo N, Sugawara Y, Imamura H, Sano K, Makuuchi M. Tailoring the type of donor hepatectomy for adult living donor liver transplantation. Am J Transplant. 2005; 5:1694-1703.

5. Sugawara Y, Makuuchi M, Sano K, Imamura H, Kaneko J, Ohkubo T, Matsui Y, Kokudo N. Vein reconstruction in modified right liver graft for living donor liver transplantation. Ann Surg. 2003; 237:180-185.

6. Gedaly R, Clifford TM, McHugh PP, Jeon H, Johnston TD, Ranjan D. Prevalent immunosuppressive strategies in liver transplantation for hepatitis C: Results of a multi-center international survey. Transpl Int. 2008; 21:867-872.

7. Sugawara Y, Makuuchi M, Kaneko J, Ohkubo T, Imamura H, Kawarasaki H. Correlation between optimal tacrolimus doses and the graft weight in living donor liver transplantation. Clin Transplant. 2002; 16:102-106.

8. First MR. First clinical experience with the new oncedaily formulation of tacrolimus. Ther Drug Monit. 2008; 30:159-166.

9. Eisen SA, Miller DK, Woodward RS, Spitznagel E, Przybeck TR. The effect of prescribed daily dose frequency on patient medication compliance. Arch Intern Med. 1990; 150:1881-1884.

(Received June 21, 2011; Revised July 28, 2011; Accepted Auguest 4, 2010) 